\title{
Exposição ao tabagismo passivo na gestação e suas consequências na amamentação: uma revisão sistemática
}

\author{
Exposure to secondhand smoke during pregnancy and its consequences on breastfeeding: a \\ systematic review
}

Exposición al humo de segunda mano durante el embarazo y sus consecuencias sobre la lactancia: una revisión sistemática

José William Araújo do Nascimento ORCID: https://orcid.org/0000-0002-1844-1117

Universidade Federal de Pernambuco, Brasil E-mail: jwan@cin.ufpe.br

Érica Lays Leite Pires

ORCID: https://orcid.org/0000-0003-2620-3239 Faculdade Maurício de Nassau, Brasil

E-mail: erica.lays@ @otmail.com

Karoliny Alves Pereira

ORCID: https://orcid org/0000-0001-7371-8687

Centro Universitário de João Pessoa, Brasil

E-mail: karolinyalvespereira@gmail.com

Glayse Xavier Gonçalves dos Santos ORCID: https://orcid.org/0000-0003-4557-4983 Faculdade Alpha, Brasil

E-mail: glayse.xavier@gmail.com

Simone Pereira da Silva

ORCID: https://orcid.org/0000-0002-5681-4527 Faculdade Alpha, Brasil E-mail: simonepereira149@gmail.com

Clarice Ester Pinto dos Passos Lins ORCID: https://orcid.org/0000-0001-5212-3502 Universidade Católica de Pernambuco, Brasil E-mail: Claricelins7@gmail.com

Lucas Mariano da Silva Barbosa ORCID: https://orcid.org/0000-0002-9264-8810 Universidade Católica de Pernambuco, Brasil E-mail: lucas.2019207491@unicap.br

Amanda da Silva Ferreira ORCID: https://orcid.org/0000-0001-6470-7521 Universidade Católica de Pernambuco, Brasil E-mail: amanda19ferreira@hotmail.com

Bárbara Barbosa Brandão ORCID: https://orcid.org/0000-0002-1145-3895 Universidade Católica de Pernambuco, Brasil E-mail: babbibbrandao21@gmail.com

Delziane Francisca Ribeiro Nascimento ORCID: https://orcid.org/0000-0003-1651-2150 Universidade Federal de Pernambuco, Brasil E-mail: delziane.ribeiro@ufpe.br

Andreza Pereira dos Santos ORCID: https://orcid.org/0000-0002-9265-6570 Universidade Católica de Pernambuco, Brasil

E-mail: andreza_pereira@outlook.com.br

Dário César de Oliveira Conceição ORCID: https://orcid.org/0000-0002-0543-2764 Faculdade Santíssima Trindade, Brasil E-mail: dariodeoliveira89@gmail.com

\section{Resumo}

Objetivo: Identificar as consequências na amamentação decorrentes da exposição ao tabagismo passivo na gestação. Metodologia: Revisão sistemática da literatura baseada no método Preferred Reporting Items for Systematic Reviews 
e Meta-Analysis (PRISMA), realizada nas bases de dados eletrônicas ScieLo, PubMed, Scopus e Web of Science, por meio dos seguintes descritores: "secondhand smoke", "pregnancy", "pregnant women" e "breastfeeding". Foram incluídos artigos completos, disponíveis em português, inglês ou espanhol, publicados entre janeiro de 2010 a outubro de 2021 e que possuem significância estatística aplicada nos resultados. Resultados: Sete artigos compuseram a amostra final deste estudo. Todas as pesquisas eram de coorte prospectivo, apresentando nível de evidência III (moderado). Grande parte das gestantes foram expostas ao fumo do tabaco em suas residências, reiterando a informação de que a fumaça dos cigarros poderia ser proveniente de familiares próximos. A exposição materna ao tabagismo passivo durante a gravidez foi significativamente associada a um aumento na interrupção de qualquer amamentação antes dos seis meses. Além disso, a exposição durante a gravidez foi associada a uma menor duração da amamentação e uma menor prevalência de amamentação. Conclusão: Verificou-se que a exposição ao tabagismo passivo durante a gravidez foi associada à interrupção ou a uma menor frequência do período de amamentação, tanto no aleitamento materno exclusivo quanto nos demais períodos, em comparação com a ausência de exposição ao tabagismo passivo durante a gravidez. Porém, pesquisas de maior qualidade são fortemente recomendadas a fim de identificar maiores resultados.

Palavras-chave: Aleitamento materno; Fumo passivo; Gravidez.

\begin{abstract}
Objective: To identify the consequences on breastfeeding resulting from exposure to passive smoking during pregnancy. Methodology: Systematic literature review based on the Preferred Reporting Items for Systematic Reviews and Meta-Analysis (PRISMA) method, carried out in the electronic databases ScieLo, PubMed, Scopus and Web of Science, using the following descriptors: "secondhand smoke", "pregnancy", "pregnant women" and "breastfeeding". Full articles, available in Portuguese, English or Spanish, published between January 2010 and October 2021 and which have statistical significance applied to the results were included. Results: Seven articles made up the final sample of this study. All studies were prospective cohorts, presenting level of evidence III (moderate). Most of the pregnant women were exposed to tobacco smoke in their homes, reiterating the information that cigarette smoke could come from close family members. Maternal exposure to passive smoking during pregnancy was significantly associated with an increase in stopping any breastfeeding before six months. In addition, exposure during pregnancy was associated with a shorter duration of breastfeeding and a lower prevalence of breastfeeding. Conclusion: It was found that exposure to passive smoking during pregnancy was associated with interruption or a lower frequency of breastfeeding, both in exclusive breastfeeding and in other periods, compared to the absence of exposure to passive smoking during pregnancy. pregnancy. However, higher quality research is strongly recommended in order to identify greater results.
\end{abstract}

Keywords: Breast feeding; Passive smoke; Pregnancy.

\title{
Resumen
}

Objetivo: Identificar las consecuencias sobre la lactancia derivadas de la exposición al tabaquismo pasivo durante el embarazo. Metodología: Revisión sistemática de la literatura basada en el método Preferred Reporting Items for Systematic Reviews and Meta-Analysis (PRISMA), realizada en las bases de datos electrónicas ScieLo, PubMed, Scopus y Web of Science, utilizando los siguientes descriptores: "humo de segunda mano", "embarazo", "mujeres embarazadas" y "lactancia materna". Se incluyeron artículos completos, disponibles en portugués, inglés o español, publicados entre enero de 2010 y octubre de 2021 y que tuvieran significación estadística aplicada a los resultados. Resultados: Siete artículos conformaron la muestra final de este estudio. Todos los estudios fueron cohortes prospectivas, con nivel de evidencia III (moderado). La mayoría de las gestantes estuvieron expuestas al humo del tabaco en sus domicilios, reiterando la información de que el humo del cigarro podría provenir de familiares cercanos. La exposición materna al tabaquismo pasivo durante el embarazo se asoció significativamente con un aumento en la interrupción de la lactancia antes de los seis meses. Además, la exposición durante el embarazo se asoció con una duración más corta de la lactancia materna y una menor prevalencia de lactancia materna. Conclusión: Se encontró que la exposición al tabaquismo pasivo durante el embarazo se asoció con la interrupción o menor frecuencia de la lactancia, tanto en lactancia exclusiva como en otros períodos, en comparación con la ausencia de exposición al tabaquismo pasivo durante el embarazo. Sin embargo, se recomienda encarecidamente una investigación de mayor calidad para identificar mejores resultados.

Palabras clave: Lactancia materna; Humo pasivo; Embarazo.

\section{Introdução}

Globalmente, o tabagismo está associado a seis das oito principais causas de morte e o controle desta problemática é uma das metas comuns de desenvolvimento sustentável global (United Nations, 2015). Neste contexto, o tabagismo passivo é compreendido como a mistura da fumaça que emana da ponta acesa de um cigarro (fumaça lateral) e a exalada pelo fumante 
(fumaça comum). Também é referido pelo termo preferido da indústria do tabaco como Fumaça Ambiental de Cigarros (FAC) (McGhee et al., 2017). O tabagismo passivo é uma mistura complexa de mais de 4.000 produtos químicos, a maioria deles formada durante o processo de combustão, incluindo nicotina, carcinógenos e toxinas. A partir de dados de biomarcadores, fica claro que os não fumantes absorvem esses produtos químicos em quantidades suficientes para serem mensuráveis (McGhee et al., 2017).

Mais de um terço da população global são fumantes passivos e regularmente expostos aos efeitos perigosos da fumaça do tabaco. Esta exposição é responsável por aproximadamente 600 mil óbitos anualmente e aproximadamente 1\% das doenças globais em todo o mundo (WHO, 2015). O resultado de um estudo realizado em 192 países mostrou que 40\% das crianças foram expostas ao fumo passivo e 36\% foram expostas ainda no ambiente uterino (Oberg et al., 2011; Cheng et al., 2017).

Nesta perspectiva, o tabagismo materno durante a gravidez é um importante problema de saúde global, resultando em vários efeitos adversos fetais (Míguez et al., 2021). Apesar das consequências negativas bem estabelecidas, estudos epidemiológicos têm mostrado que entre 11 e 30\% das mulheres grávidas fumam ou são expostas ao fumo passivo, de tal modo que essa taxa aumenta para 50\% em amostras de alto risco, incluindo jovens, populações de baixa renda e residentes em áreas urbanas (Míguez et al., 2021).

Evidências convincentes foram publicadas sobre os efeitos causais da exposição ao tabaco durante a gravidez em várias condições reprodutivas e obstétricas, incluindo gravidez ectópica, aborto espontâneo, natimorto, patologias placentárias, parto muito prematuro e pré-termo, problemas na amamentação, baixo peso ao nascer, síndrome da morte súbita do bebê e problemas cognitivos, emocionais, temperamentais e comportamentais envolvendo a prole (Peña et al., 2012; Phelan, 2014; Huang et al., 2017).

As consequências do tabagismo passivo para o aleitamento materno por sua vez têm sido fruto de investigações científicas dado alguns resultados adversos relatados por alguns estudos experimentais, como redução dos níveis basais de prolactina e consequente diminuição da oferta de leite materno (Del Ciampo et al. 1999), mudanças na composição e sabor do leite (Napierala et al., 2016) e tendência ao desmame precoce (Kaufmann et al., 2012). Mães que foram expostas passivamente ao tabaco no período pré-natal desmamam seus bebês mais cedo do que as não fumantes, e essas mães têm maior risco de problemas (mastite) quando começam o desmame (Liu et al., 2006). Uma pesquisa realizada com 552 mulheres e verificada pela dosagem de cotinina em amostras de urina e sangue de algumas das entrevistadas, revelou que o grupo rotineiramente exposto ao tabaco tem menor probabilidade de serem mães que amamentam (Chou et al., 2008).

Embora haja ampla evidência na literatura sobre os riscos associados ao tabagismo materno durante a gravidez, poucos estudos enfocaram os efeitos específicos na amamentação decorrentes da exposição passiva devido ao consumo de tabaco pelo companheiro da mãe e/ou outros membros da família. Desta forma, este estudo objetivou identificar as consequências na amamentação decorrentes da exposição ao tabagismo passivo na gestação.

\section{Metodologia}

Esta revisão sistemática foi conduzida com base no método de Preferred Reporting Items for Systematic Reviews and Meta-Analysis (PRISMA) (Moher et al., 2015). Salienta-se que as revisões sistemáticas são o padrão ouro para sintetizar e analisar as evidências científicas devido ao seu rigor metodológico, sendo bastante utilizados para apoiar o desenvolvimento de diretrizes de prática clínica e informar a tomada de decisão (Moher et al., 2015).

Foi seguida as seguintes etapas, idealizada por Pati \& Lorusso (2018): 1) Definição da questão norteadora e objetivos da pesquisa; 2) Estabelecimento dos critérios de inclusão e exclusão/ amostragem dos estudos; 3) Busca na literatura; 4)

Categorização e análise dos estudos; 5) Apresentação e discussão dos resultados da amostra e; 6) Apresentação e síntese do 
conhecimento. A questão norteadora deste estudo incide em: "Quais consequências a exposição ao tabagismo passivo na gestação pode ocasionar na amamentação?"

A fim de responder a esta questão norteadora, em uma busca sistemática, quatro bancos de dados eletrônicos foram utilizados, incluindo, Scientific Electronic Library Online (ScieLo), Nacional Institute of Medicine (NIH-PUBMED), Scopus e Web of Science, no período de outubro a dezembro de 2021. Realizou-se consulta ao Medical Subject Headings (MeSH) para seleção dos descritores a serem utilizados, a saber: "secondhand smoke", "pregnancy", "pregnant women" e "breastfeeding".

Foram incluídos estudos com textos completos, disponíveis em português, inglês ou espanhol, publicados entre janeiro de 2010 a outubro de 2021 e que possuem significância estatística aplicada nos resultados. Os critérios de exclusão abrangeram estudos que tinham como amostra mães que antes fumavam e paravam de fumar antes de gravidez, gestantes que fumaram durante a gestação, estudos não comparativos, artigos duplicados nas bases de dados (duplicatas), estudos de revisão e pesquisas com temática não relevante a questão norteadora da pesquisa.

Para garantir o registro do conjunto de informações relevantes ao tema utilizou-se um formulário adaptado, elaborado para o presente estudo, que adotou as orientações fornecidas pela Cochrane Collaboration (Higgins \& Green, 2011) no que se refere ao conteúdo e à estrutura.

Após o levantamento nas bases de dados, os estudos foram submetidos às etapas de identificação e análise dos dados. Para o desenvolvimento dessa fase, foi utilizada uma tabela do Google Sheets compartilhada entre os pesquisadores do presente trabalho. A tabela contém os seguintes campos na seguinte ordem: ID (um contador crescente para identificação do artigo), autores, título, ano de Publicação, local de Publicação, base de dados e link da publicação. Cada pesquisador foi responsável por bases de dados diferentes, dividido de forma com que cada um adicionasse um número semelhante de artigos à tabela.

A última atividade dessa fase foi a remoção de duplicatas de artigos, ou seja, a remoção de artigos que foram retornados por mais de uma base de dados para que permaneça apenas uma instância do mesmo na planilha. A tabela resultante dessa etapa contém o conjunto de artigos selecionados que é utilizado na segunda etapa. Nesta segunda fase, etapa de triagem, todos os títulos e resumos dos artigos foram examinados com base nos objetivos principais de selecionar estudos relevantes pelos revisores. Posteriormente, os textos completos de estudos relevantes foram examinados minuciosamente por revisores.

Assim sendo, os artigos filtrados nas bases de dados foram analisados (n: 1.367), sendo excluído de imediato os duplicados, considerando-se apenas o primeiro a ser identificado. Desta forma, após 257 duplicatas serem removidas, 1.110 estudos foram analisados quanto a seus títulos e resumos, sendo excluídos 980 estudos após incorporação dos critérios de elegibilidade. Na etapa seguinte, os estudos elegíveis (n: 130) foram lidos na íntegra por pesquisadores de forma independente. Após as análises e resolução das discrepâncias entre as avaliações, sete artigos foram incluídos na amostra final do estudo para a extração dos dados. Um resumo das etapas realizadas encontra-se disposto na Figura. 1. 
Figura 1: Fluxograma do processo de seleção do estudo, Recife (PE), Brasil, 2022.

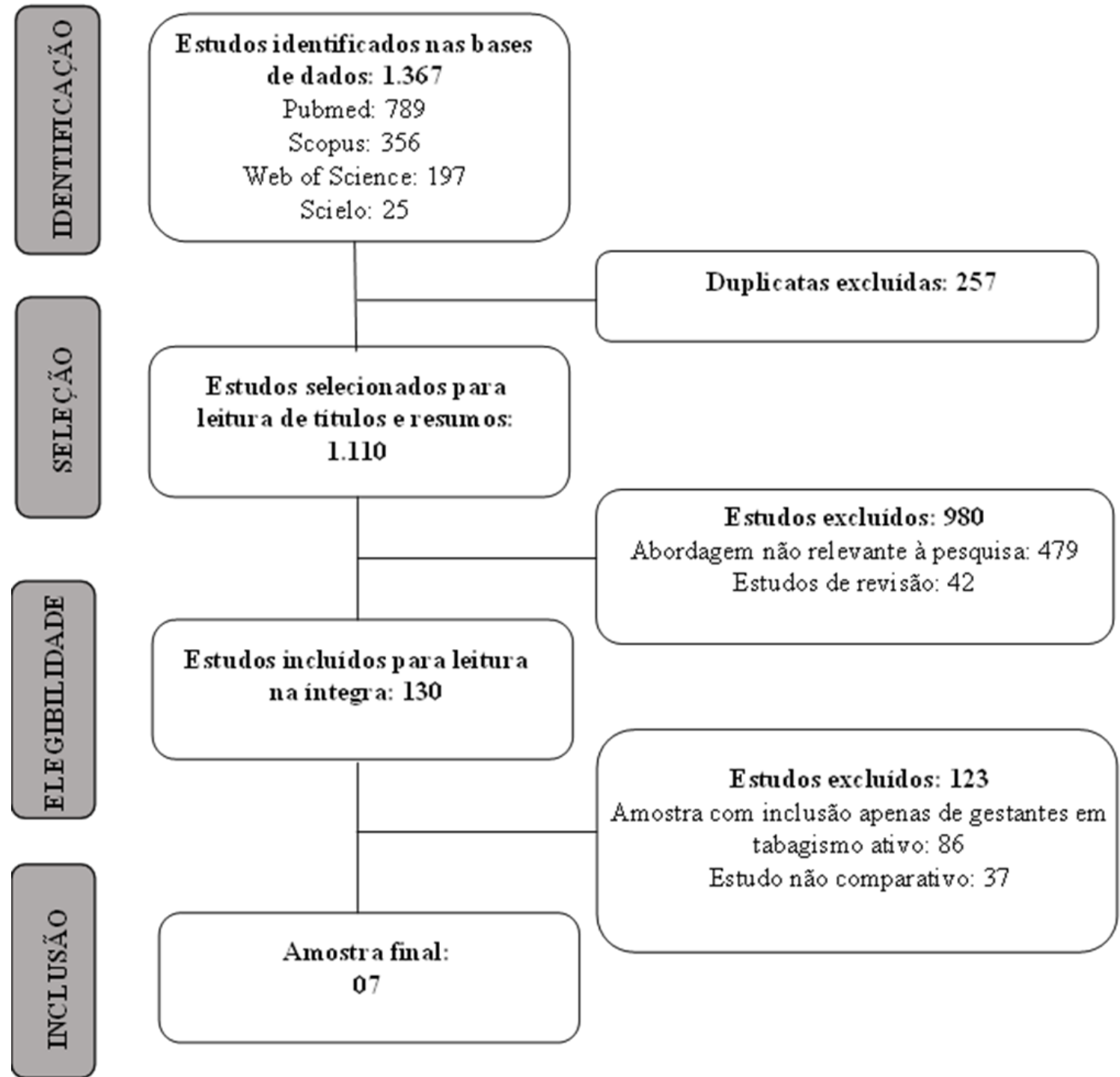

Fonte: Autores.

Os artigos selecionados foram submetidos à classificação do nível de evidência, a partir do instrumento de Classificação Hierárquica das Evidências para Avaliação dos Estudos (Stillwell et al., 2010). Segundo esta classificação, os níveis I e II são considerados evidências fortes, III e IV moderadas e V a VII fracas.

Este estudo preservou os aspectos éticos de tal forma que todos os autores das publicações analisadas foram referenciados apropriadamente, mediante a Lei de Direitos Autorais nº 9.610 de 19 de Fevereiro de 1998 (Brasil, 1998).

\section{Resultados}

Sete publicações compuseram a amostra final deste estudo. A Tabela 1 evidencia as características das pesquisas incluídas. Constata-se que a maior frequência de publicação destes artigos foi dos anos de 2013 (n: 02) e 2019 (n: 02), enquanto o país de origem mais frequentemente listado tenha sido os EUA (n: 03). Referindo-se a tipologia das pesquisas é possível identificar que prevaleceu o estudo de coorte prospectivo (n: 07), com um nível de evidência considerado moderado (III). 
Tabela 1: Características gerais dos estudos incluídos na amostra, Recife (PE), Brasil, 2022.

\begin{tabular}{|c|c|c|c|c|c|}
\hline ID & Autores (ano) & País do estudo & Periódico (Qualis) & $\begin{array}{l}\text { Tipo de estudo } \\
\text { (NE*) }\end{array}$ & Objetivos \\
\hline 1 & $\begin{array}{l}\text { Kwok et al. } \\
\qquad(2010)\end{array}$ & Hong Kong & Pediatrics & $\begin{array}{l}\text { Estudo de coorte } \\
\text { prospectivo (III) }\end{array}$ & $\begin{array}{l}\text { Examinar a exposição pré-natal ou pós-natal ao fumo } \\
\text { passivo em filhos de mães não fumantes. }\end{array}$ \\
\hline 2 & $\begin{array}{l}\text { Lemke et al. } \\
\qquad(2013)\end{array}$ & EUA & $\begin{array}{l}\text { Annals of Allergy, } \\
\text { Asthma, \&. } \\
\text { Immunology }\end{array}$ & $\begin{array}{l}\text { Estudo de coorte } \\
\text { prospectivo (III) }\end{array}$ & $\begin{array}{l}\text { Avaliar a propensão de resultados pós-natais adversos } \\
\text { em decorrência do tabagismo passivo. }\end{array}$ \\
\hline 3 & $\begin{array}{l}\text { Salama et al. } \\
\qquad(2013)\end{array}$ & Egito & $\begin{array}{l}\text { Indian Journal of } \\
\text { Clinical } \\
\text { Biochemistry }\end{array}$ & $\begin{array}{l}\text { Estudo de coorte } \\
\text { prospectivo (III) }\end{array}$ & $\begin{array}{l}\text { Determinar o nível de cotinina, enolase específica do } \\
\text { neurônio, caderina epitelial humana solúvel (sE- } \\
\text { caderina) e óxido nítrico na sucção traqueal de neonatos } \\
\text { expostos e não expostos ao tabagismo no pré-natal. }\end{array}$ \\
\hline 4 & $\begin{array}{l}\text { Baheiraei et al. } \\
\qquad(2014)\end{array}$ & Irã & $\begin{array}{l}\text { Journal of Public } \\
\text { Health (Germany) }\end{array}$ & $\begin{array}{l}\text { Estudo de coorte } \\
\text { prospectivo (III) }\end{array}$ & $\begin{array}{l}\text { Avaliar o efeito da exposição materna ao fumo passivo } \\
\text { na duração do aleitamento materno exclusivo. }\end{array}$ \\
\hline 5 & $\begin{array}{l}\text { Moore et al. } \\
\qquad(2017)\end{array}$ & EUA & Pediatric Obesity & $\begin{array}{l}\text { Estudo de coorte } \\
\text { prospectivo (III) }\end{array}$ & $\begin{array}{l}\text { Examinar a interação entre a exposição ao fumo passivo } \\
\text { e exclusividade da amamentação na adiposidade aos } 5 \\
\text { meses de idade. }\end{array}$ \\
\hline 6 & $\begin{array}{l}\text { Moore et al. } \\
\qquad(2019)\end{array}$ & EUA & $\begin{array}{l}\text { International } \\
\text { Journal of Obesity }\end{array}$ & $\begin{array}{l}\text { Estudo de coorte } \\
\text { prospectivo (III) }\end{array}$ & $\begin{array}{l}\text { Explorar as associações entre a exposição fetal ao } \\
\text { tabagismo materno ativo e passivo com a composição } \\
\text { corporal no nascimento e as trajetórias de crescimento do } \\
\text { IMC até os } 3 \text { anos de idade. }\end{array}$ \\
\hline 7 & Lee et al. (2019) & Coreia do Sul & $\begin{array}{l}\text { Environmental } \\
\text { Health }\end{array}$ & $\begin{array}{l}\text { Estudo de coorte } \\
\text { prospectivo (III) }\end{array}$ & $\begin{array}{l}\text { Investigar a associação entre a exposição pré-natal ao } \\
\text { tabagismo passivo e o neurodesenvolvimento dos recém- } \\
\text { nascidos. }\end{array}$ \\
\hline
\end{tabular}

Nota: *NE - Nível de evidência. Fonte: Autores.

Conforme a Tabela 2, que apresenta os desfechos encontrados nos estudos acerca das consequências do tabagismo passivo na amamentação, verifica-se que quanto à população estudada, os estudos incluídos avaliaram um total de 5.895 mulheres gestantes que foram expostas ao tabagismo passivo no período pré-natal, com uma idade média variando de 22,56 a 27,43 anos. Grande parte destas gestantes foram expostas ao fumo do tabaco em suas residências (dois estudos não incluíram esta informação - ID3 e ID7), reiterando a informação de que a fumaça dos cigarros poderia ser proveniente de familiares próximos.

Ainda na Tabela 2 é possível analisar que quatro estudos se concentraram em avaliar o tempo e a frequência do aleitamento materno em qualquer período (ID1, ID2, ID3 e ID7). O tempo de medição dos resultados variou de 1 mês até 3 meses, de tal forma que dois estudos não relataram o período (ID2 e ID7), sendo um viés importante para interpretação dos resultados. Apesar desta inconsistência, estes estudos relataram chances significativas de interrupção do aleitamento materno após a mãe ter sido exposta passivamente ao tabaco no período pré-natal, com uma frequência de ausência de oferta do aleitamento variando de $11,1 \%$ a $86 \%$.

Três pesquisas avaliaram resultados inerentes apenas ao período de aleitamento materno exclusivo (até o sexto mês) - 
(ID4, ID5 e ID6). Ambos os estudos definiram a exposição ao tabagismo em casa e os pontos de medição foram seis meses após o parto, considerando, desta forma, a presença de um rigor metodológico destes estudos quanto a este quesito. Constata-se que estas pesquisas apresentaram análises estatísticas significantes, indicando chances entre 2 a 3 vezes de apresentar uma redução significativa do período e frequência do aleitamento materno exclusivo.

Tabela 2: Principais consequências da exposição ao tabagismo passivo no período pré-natal na amamentação, Recife (PE),

Brasil, 2022.

\begin{tabular}{|c|c|c|c|c|c|}
\hline \multirow[t]{2}{*}{ ID } & \multicolumn{2}{|c|}{$\begin{array}{l}\text { Características das gestantes expostas } \\
\text { ao tabagismo passivo }\end{array}$} & \multirow{2}{*}{$\begin{array}{l}\text { Local de } \\
\text { exposição }\end{array}$} & \multirow{2}{*}{$\begin{array}{l}\text { Tempo de } \\
\text { medição de } \\
\text { resultado após } \\
\quad \text { o parto }\end{array}$} & \multirow[t]{2}{*}{ Consequências do tabagismo passivo na amamentação } \\
\hline & $\mathbf{N}$ & $\begin{array}{l}\text { Idade média } \pm \\
\text { DP* }\end{array}$ & & & \\
\hline 1 & 4389 & $-* *$ & Residência & 3 meses & $\begin{array}{l}86 \% \text { não conseguiram amamentar em qualquer período pós- } \\
\text { parto, apontando para relação entre a exposição a uma menor } \\
\text { propensão de amamentação }(\mathrm{p}<0,001)\end{array}$ \\
\hline 2 & 257 & 24 & Residência & ${ }^{* *}$ & $\begin{array}{l}53 \% \text { das mães expostas não conseguiram amamentar os } \\
\text { neonatos, com uma significância estatística de } \mathrm{p}<0,001\end{array}$ \\
\hline 3 & 100 & $22.56 \pm 3.9$ & $-^{* *}$ & 1 mês & $\begin{array}{l}\text { Diminuição significativa na continuação do aleitamento } \\
\text { materno no grupo exposto em comparação ao não exposto }(\mathrm{p}= \\
0,008)\end{array}$ \\
\hline 4 & 170 & $27.43 \pm 4.34$ & Residência & 6 meses & $\begin{array}{l}\text { Redução significativa no aleitamento materno exclusivo } \\
\text { durante um período de } 6 \text { meses }(\mathrm{p}<0,001) \text {. Mães fortemente } \\
\text { expostas }\left(\mathrm{OR}^{+}=2,22, \mathrm{IC}^{\theta} 95 \%: 1,45-3,39\right) \text { e mães levemente } \\
\text { expostas }\left(\mathrm{OR}^{+}=1,65 \text {, IC }{ }^{\theta} 95 \%: 1,25-2,42\right) \text { foram mais } \\
\text { propensas a interromper a amamentação exclusiva mais cedo } \\
\text { em comparação com mulheres não expostas. }\end{array}$ \\
\hline 5 & 129 & 25 & Residência & 6 meses & $\begin{array}{l}\text { A combinação de exposição ao fumo passivo e falta de } \\
\text { aleitamento materno exclusivo foi associada ao aumento da } \\
\text { adiposidade aos } 5 \text { meses de idade. Por exemplo, dentro dos } \\
\text { estratos não amamentados exclusivamente, a exposição ao } \\
\text { fumo passivo foi associada ao aumento da massa gorda }(0,1 \mathrm{~kg} \text {; } \\
\text { IC } 95 \% \text { : } 0,0-0,2 ; \mathrm{P}=0,05) \text {. Por outro lado, dentro dos estratos } \\
\text { amamentados exclusivamente, não houve praticamente } \\
\text { nenhuma diferença na massa gorda entre bebês expostos e não } \\
\left.\text { expostos (coeficiente: }-0,1 ; \mathrm{IC}^{\theta} 95 \% \text { : }-0,3-0,1 ; \mathrm{P}=0,25\right) \text {. }\end{array}$ \\
\hline 6 & 130 & $24 \pm 6$ & Residência & 6 meses & $\begin{array}{l}75 \% \text { do grupo exposto ofertou um aleitamento materno } \\
\text { exclusivo por menos de } 5 \text { meses; este grupo apresentou maior } \\
\text { chance de ofertar por menos tempo o aleitamento materno } \\
\text { exclusivo }(\mathrm{p}<0,001)\end{array}$ \\
\hline 7 & 720 & $-* *$ & $-* *$ & $-^{* *}$ & $\begin{array}{l}35,7 \% \text { das mães ofertou o leite materno até os } 6 \text { meses (não } \\
\text { exclusivo) e } 11,1 \% \text { não ofertaram em nenhum momento (p < } \\
0,001 \text { ). }\end{array}$ \\
\hline
\end{tabular}

Nota: *DP - Desvio Padrão; ** Não relatado; +OR - Odds Ratio (razão de chance); $\theta$ IC - Intervalo de Confiança. Fonte: Autores. 


\section{Discussão}

Este estudo analisou a produção científica a respeito das consequências da exposição ao tabagismo passivo no período pré-natal na amamentação, a fim de fomentar uma discussão científica mais abrangente acerca desta problemática.

Quanto ao desenho metodológico das publicações analisadas, prevaleceu os estudos de coorte prospectivos, que possuem um nível de evidência moderado conforme a classificação dos níveis de evidências de estudos científicos explicitados na metodologia. Em um estudo de coorte, um desfecho ou população de estudo livre de doença é primeiro identificado pela exposição ou evento de interesse e seguido no tempo até que ocorra a doença ou o desfecho de interesse. Como a exposição é identificada antes do desfecho, os estudos de coorte têm uma estrutura temporal para avaliar a causalidade e, portanto, têm o potencial de fornecer as evidências científicas mais fortes (Everitt \&Palmer, 2005; Elwood, 2007).

Porém verificou-se que os estudos de coorte prospectivos realizados apresentaram algumas inconsistências como amostra reduzida e baixo período de acompanhamento das mães após o parto, características estas, inerentes para um bom seguimento de uma pesquisa desta natureza. Neste sentido, apesar dos resultados favoráveis, estudos mais robustos precisam ser desenvolvidos a fim de tornar mais claro a discussão dos resultados identificados.

Acerca das consequências da exposição das gestantes ao tabagismo passivo na amamentação, os resultados de nosso estudo demonstraram uma associação significativa entre esta exposição com uma diminuição na quantidade e frequência da amamentação. Todos os estudos analisados foram enfáticos ao trazer estes resultados em comparação com gestantes não expostas ao tabagismo.

Nesta perspectiva, há um número de explicações possíveis para um efeito adverso da fumaça de cigarro na amamentação; estes incluem um evento fisiológico de nicotina no sistema hormonal da mãe ou diretamente sobre as mamas (Napierala et al., 2016). Como o tabagismo passivo opera através da mãe, ele pode influenciar o aleitamento materno por um evento sobre a produção de prolactina. Eventualmente, esta exposição pode talvez mudar o sabor do leite humano, tornando-o menos saboroso para as crianças. Além disso, o tabagismo passivo aumenta a frequência de infecções respiratórias recorrentes nas crianças, podendo afetar o seu desejo por alimentos e enfraquecer a estimulação dos mamilos e reduzir a produção de leite materno (Miller et al., 2004; Liu et al., 2006).

Já se sabe que o fumo ou a nicotina parenteral estão associados a baixas concentrações de prolactina (Shenassa et al., 2015). Este hormônio é importante para a homeostase metabólica e está associada à glândula mamária em lactação, por aumentar as proteínas do leite, lactose e lipídios (Ben-Jonathan et al., 2006).

O tabagismo passivo apresenta um risco maior do que a fumaça da corrente principal porque o este tipo de tabagismo é uma combinação de fumaça da corrente principal, aquela que é exalada pelo fumante e a fumaça do fluxo lateral, aquela fumaça que é emitida do cigarro entre as tragadas. A fumaça da corrente lateral inclui um maior número de produtos químicos do que a fumaça da corrente principal. Portanto, durante a exposição ao fumo passivo, a nicotina ou outros produtos químicos são inalados, resultando na inibição da liberação de prolactina (Shenassa et al., 2015). Além destes fatores preponderantes, como a nicotina leva à vasoconstrição, uma diminuição do fluxo sanguíneo para a mama pode reduzir os níveis de ocitocina circulante, o que reduziria o leite materno disponível para a criança (Amir, 2001; Bahadori et al., 2013).

Os achados deste estudo ainda revelam que a duração do aleitamento materno entre mulheres expostas ao fumo passivo tende a ser menor do que o recomendado pela World Organization Health (WHO). A recomendação é que os lactentes precisassem de aleitamento materno exclusivo até os seis meses e depois continuassem por até dois anos ou mais e complementado com alimentos sólidos, isto porque a amamentação fornece nutrição essencial para o crescimento do lactente e é uma forma fácil de proporcionar os efeitos protetores (WHO, 2022). Além de atender às necessidades nutricionais do lactente, o aleitamento materno reduz a mortalidade, morbidade e hospitalização de crianças por doenças infecciosas, como 
diarreia, pneumonia e otite (Victora et al., 2016; Buccini et al., 2017).

\section{Conclusão}

Verificou-se que a exposição ao tabagismo passivo durante a gravidez foi associada à interrupção ou a uma menor frequência do período de amamentação, tanto no aleitamento materno exclusivo quanto nos demais períodos, em comparação com a ausência de exposição ao tabagismo durante a gravidez. Porém, pesquisas de maior qualidade são fortemente recomendadas a fim de identificar maiores resultados.

Salienta-se que as indicações de que a exposição ao tabagismo passivo pode afetar a duração da amamentação implica que mais pesquisas são necessárias para informar as estratégias de saúde pública que poderiam prevenir os efeitos adversos desta exposição na saúde materno-infantil. Dados os benefícios da amamentação, uma campanha de prevenção antitabagismo poderia ser uma estratégia a ser adotada, a partir da orientação sobre o impacto da exposição ao fumo passivo na saúde.

\section{Referências}

Alves, T. O., Nunes, R. L. N., Sena, L. H. A., Alves, F. G., Souza, A. G. S., Salviano, A. M., et al (2021). Gestação de alto risco: epidemiologia e cuidados, uma revisão de literatura. BJRH. 4(4), 14860-14872.

Amir, L. H. (2001). Maternal smoking and reduced duration of breastfeeding: a review of possible mechanisms. Early Hum Dev. 64(1), 45-67.

Bahadori, B., Riediger, N. D., Farrell, S. M., Uitz, E. \& Moghadasian, M. F. (2013). Hypothesis: smoking decreases breast feeding duration by suppressing prolactin secretion. Med. Hypotheses. 81(4), 582-586.

Baheiraei, A., Ghafoori, F., Rahimi Foroushani, A. \& Nedjat, S. (2014). The effects of maternal exposure to second-hand smoke on breastfeeding duration: A prospective cohort study. Journal of Public Health (Germany). 22, 13-22.

Ben-Jonathan, N., Hugo, E. R., Brandebourg, T. D. \& LaPensee, C. R. (2006). Focus on prolactin as a metabolic hormone. Trends Endocrinol Metab. 17(3), $110-116$.

Buccini, G. S., Pérez-Escamilla, R., Paulino, L. M., Araújo, C. L. \& Venâncio, S. I. (2017). Pacifier use and interruption of exclusive breastfeeding: Systematic review and meta-analysis. Matern Child Nutr. 13(3), e12384.

Cheng, K. W., Chiang, W. L. \& Chiang, T. L. (2017). In utero and early childhood exposure to secondhand smoke in Taiwan: A population-based birth cohort study. BMJ Open. 7(6), 1-10.

Chou, S. Y., Hsu, H. H., Kuo, H. H. \& Kuo, H. W. (2008). Association between exposure to environmental tobacco smoke (ETS) and breastfeeding behavior. Acta Paediatr. 97, 76-80.

Elwood, M. (2007). Critical Appraisal of Epidemiological Studies and Clinical Trials. 3rd Ed Oxford University Press; Oxford, 1-570.

Everitt, B. S. \& Palmer, C. R. (2005). Encyclopaedic Companion to Medical Statistics. Hodder Arnold; London.

Del Ciampo, L. A., Almeida, C. A. \& Ricco, R. G. (1999). Passive smoking in early life. Pediatria (São Paulo). 21, 15-20.

Higgins, J. \& Green, S. (2011). Cochrane handbook for systematic reviews of interventions version 5.1.0. Chichester: John Wiley \& Sons.

Kaufmann, C. C., Albernaz, E. P., Silveira, R. B., Silva, M. B. \& Mascarenhas, M. L. (2012). Alimentação nos primeiros três meses de vida de bebês de uma coorte de Pelotas, RS, Brasil. Rev Paul Pediatr. 30, 157-65.

Kwok, M. K., Schooling, C. M., Lam, T. H. \& Leung, G. M. (2010). Paternal smoking and childhood overweight: evidence from the Hong Kong “Children of 1997'. Pediatrics. 126, e46-56.

Lee, M., Ha, M., Hong, Y. C., Park, H., Kim, Y., Kim, E. J., et al. (2019). Exposure to prenatal secondhand smoke and early neurodevelopment: Mothers and Children's Environmental Health (MOCEH) study. Environ Health. 18(1), 22.

Lemke, M., Hartert, T. V., Gebretsadik, T. \& Carroll, K. N. (2013). Relationship of secondhand smoke and infant lower respiratory tract infection severity by familial atopy status. Annals of Allergy, Asthma, \&. Immunology. 110, 433-437.

Liu, J., Rosenberg, K. D. \& Sandoval, A. P. (2006). Breastfeeding duration and perinatal cigarette smoking in a population-based cohort. Am. J. Public Health. 96, 309-314.

McGhee, S. M. \& Hedley, A. J. (2017). International Encyclopedia of Public Health (Second Edition). Reference Module in Biomedical Sciences. 455-458.

Míguez, M. C. \& Pereira, B. (2021). Effects of active and/or passive smoking during pregnancy and the postpartum period. Anales de Pediatría (English Edition). 95(4), 222-232. 
Research, Society and Development, v. 11, n. 2, e35111225626, 2022

(CC BY 4.0) | ISSN 2525-3409 | DOI: http://dx.doi.org/10.33448/rsd-v11i2.25626

Miller, R. I., GarWnkel, R., Horton, M., Camann, D., Perera, F. P., Whyatt, R., et al. (2004). Polycyclic aromatic hydrocarbons, environmental tobacco smoke, and respiratory symptoms in an inner-city birth cohort. Chest. 126(4), 1071-1078

Moher, D., Shamseer, L., Clarke, M.; Ghersi, D., Liberati, A., Petticrew, M., et al (2015). Preferred reporting items for systematic review and meta-analysis protocols (PRISMA-P) 2015 statement. Syst. Rev. 4(1), 1-9.

Moore, B. F., Sauder, K. A., Starling, A. P., Ringham, B. M., Sorte, D.H. \& Dabelea D. (2017). Exposure to secondhand smoke, exclusive breastfeeding and infant adiposity at age 5 months in the Healthy Start study. Pediatr Obes. 12(1), 111-119.

Moore, B. F., Starling, A. P., Magzamen, S., Harrord, C. S., Allshouse, W. B., Adgate, J. L., et al. (2019). Fetal exposure to maternal active and secondhand smoking with offspring early-life growth in the Healthy Start study. Int J Obes (Lond). 43(4), 652-662.

Napierala, M., Mazela, J., Merritt, T. A. \& Florek, E. (2016). Tobacco smoking and breastfeeding: Effect on the lactation process, breast milk composition and infant development. A critical review. Environmental Research. 151, 321-338.

Öberg, M., Jaakkola, M. S., Woodward, A., Peruga, A. \& Prüss-Ustün, A. (2011). Worldwide burden of disease from exposure to second-hand smoke: a retrospective analysis of data from 192 countries. Lancet. 377(9760), 139-146.

Peña, Y. P. D., Martínez, G. R., Villagrasa, M. P. S., Pérez, V. C., Andrés, L. C., Sauras, M. L. A., et al. (2012). Socio-cultural, obstetric and anthropometric characteristics of newborn children of mothers who smoke in spain. Anales de Pediatría. 76(1), 4-9.

Phelan, S. (2014). Smoking cessation in pregnancy. Obstet Gynecol Clin North Am. 41, 255-266.

Salama, R. H. M., Abdel-Aal, D. E. D. M., Eshra, D. K., Nagieb, S. \& Arief, A. F. (2013). Clinical and biochemical effects of environmental tobacco smoking on pregnancy outcome. Indian Journal of Clinical Biochemistry. 28, 368-373.

Shenassa, E. D., Wen, X., \& Braid, S. (2015). Exposure to Tobacco Metabolites via Breast Milk and Infant Weight Gain: A Population-Based Study. J. Hum. Lact.

Stillwell, S., Fineout-Overholt, E., Melnyk, B.M. \& Wiliamson, K.M. (2010). Evidence- based practice: step by step. Am J Nurs; $110(5), 41-47$.

United Nations. (2015). Transforming our World: The 2030 Agenda for Substainable Development. Department of Economic and Social Affairs Sustainable Development, United Nations.

Victora, C. G., Aluísio, J. D., Barros, A. J. D., França, G. V. A., Horton, S., Krasevec, J., et al. (2016). Breastfeeding in the 21st century: Epidemiology, mechanisms, and lifelong effect. Lancet. 387, 475-490.

World Health Organizaton (WHO). (2015). Second-hand smoke. The global health observatory: WHO.

World Health Organizaton (WHO). (2022). Breastfeeding: recommendations. Health topics, WHO. 